\title{
The Effect of Experimental Periodontitis on the Serum Levels of Leptin and IL-18 in Insulin-Treated Diabetic Rats
}

\author{
Eudoxie Pepelassi ${ }^{1,}$, , Ioanna Xynogala ${ }^{1}$, Despina Perrea ${ }^{2}$, Alkistis Pantopoulou ${ }^{2}$, \\ George Agrogiannis ${ }^{3}$, Ioannis Vrotsos ${ }^{1}$ \\ ${ }^{1}$ Department of Periodontology, School of Dentistry, National and Kapodistrian University of Athens, Athens, Greece \\ ${ }^{2}$ Laboratory for Experimental Surgery \& Surgical Research "N. S. Christeas”, School of Medicine, National and Kapodistrian University of \\ Athens, Athens, Greece \\ ${ }^{3} 1^{\text {st }}$ Department of Pathology, School of Medicine, National and Kapodistrian University of Athens, Athens, Greece
}

\section{Email address:}

epepela@dent.uoa.gr (E. Pepelassi),yannaxyn@gmail.com (I. Xynogala),dperrea@med.uoa.gr (D. Perrea),

alk_pantopoulou@yahoo.gr (A. Pantopoulou),agrojohn@med.uoa.gr (G. Agrogiannis), ivrotsos@dent.uoa.gr (I. Vrotsos)

${ }^{*}$ Corresponding author

\section{To cite this article:}

Eudoxie Pepelassi, Ioanna Xynogala, Despina Perrea, Alkistis Pantopoulou, George Agrogiannis, Ioannis Vrotsos. The Effect of Experimental Periodontitis on the Serum Levels of Leptin and IL-18 in Insulin-Treated Diabetic Rats. American Journal of Internal Medicine. Vol. 8, No. 3, 2020, pp. 101-106. doi: 10.11648/j.ajim.20200803.12

Received: March 8, 2020; Accepted: April 3, 2020; Published: April 30, 2020

\begin{abstract}
The aim of this study in rats was to assess the effect of ligature-induced periodontitis on insulin-treated type 1 diabetes in terms of serum levels of leptin and interleukin-18 (IL-18). Twenty five male Wistar rats were studied, out of 40 initially used: (1) 14 rats with insulin-treated diabetes (control, DI) and (2) 11 rats with combination of insulin-treated diabetes and periodontitis (test, DIP). For all rats, type 1 diabetes was streptozotocin-induced and insulin treatment started on day 5. For DIP group, periodontitis was induced by ligation on day 16. Serum levels of leptin and IL-18 were assessed on days 16 (baseline) and 77 (final) by using multiplex immunoassay. All rats were sacrificed on day 77 (end of the study). Insulin treatment in the newly-induced diabetes significantly increased serum leptin levels and significantly reduced serum IL-18 levels. In the newly-induced diabetes, the combination of the continuation of insulin treatment and periodontitis nonsignificantly increased serum leptin and IL-18 levels. Serum IL-18 levels were significantly higher for the combination of periodontitis and insulin-treated diabetes as compared to insulin-treated diabetes alone. Within its limits, this study in rats showed that experimental periodontitis induced changes in serum biomarker levels suggestive of alterations in the systemic inflammation generated by insulin-treated type 1 diabetes.
\end{abstract}

Keywords: Diabetes Mellitus, Type 1, Insulin, Periodontitis, Interleukin-18, Leptin, Biologic Markers

\section{Introduction}

Diabetes is a heterogenous group of metabolic diseases, where hyperglycemia arises from deficient insulin secretion, insulin activity or both. Chronic hyperglycemia damages tissues and organs in the long-term. The etiopathogenesis of diabetes involves processes that range from autoimmune destruction of the pancreatic b-cells resulting in insulin deficiency to disorders resulting in insulin resistance. These disorders are based on deficient insulin activity. Inadequate insulin secretion and/or reduced response to insulin lead to deficient insulin activity. Impaired insulin secretion and deficient insulin activity might coexist. Type 1 (T1) and 2 (T2) diabetes differ in pathogenesis, clinical manifestation and disease progression. The immune-mediated $\mathrm{T} 1$ diabetes results from a cellular mediated autoimmune destruction of the b-cells of the pancreas. [1]

Periodontitis is defined as "a chronic multifactorial inflammatory disease associated with dysbiotic plaque biofilms and characterized by progressive destruction of the tooth-supporting apparatus". [2] The relationship between diabetes and periodontitis has been studied mostly for $\mathrm{T} 2$ diabetes, whereas there is limited information on $\mathrm{T} 1$ diabetes and periodontitis. [3] In T2 diabetes, hyperglycemia is 
associated with adverse periodontal outcomes and severe periodontitis has negative impact on glycemic control. [4]

Biologic markers (or biomarkers) are implicated in the host immune response and diabetes. [5-10] In $\mathrm{T} 1$ diabetes, changes in serum levels of biomarkers might occur. [11-13] In periodontitis, the local inflammation might induce systemic inflammation at certain grade [14] and serum biomarker levels might be altered. [10] Advanced glycation end products, as a result of chronic hyperglycemia, are related to the increase of inflammation biomarkers, which then affect other biomarkers. [15] Leptin and interleukin-18 (IL-18) are related to $\mathrm{T} 1$ diabetes $[11,13,16,17]$ and inflammation. $[6,18]$ The impact of insulin treatment on serum biomarkers levels has been earlier addressed. [11, 16, 19] When the insulin-treated diabetes is combined with periodontitis, data on serum biomarkers level changes with insulin treatment are very limited.

The limited data on the relationship between insulintreated $\mathrm{T} 1$ diabetes and periodontitis led the authors to examine whether the systemic burden of insulin-treated T1 diabetes is altered by periodontitis. Periodontal tissue loss, time of T1diabetes onset and diagnosis, glycemic control, medication and diabetes complications might be difficult to control in studies in humans. In animal studies, the induction of experimental T1 diabetes, the initiation of insulin treatment and the induction of experimental periodontitis at specific time points prevent such limitations. The ligatureinduced periodontitis model $[20,22]$ and the streptozotocin (STZ)-induced diabetes model [20, 22, 23] have been studied in rats. The STZ-induced diabetes is $\mathrm{T} 1$ diabetes. Rats were studied in the present experiment.

The following hypotheses were examined in this study: (1) insulin treatment affects the circulating biomarkers in experimentally-induced $\mathrm{T} 1$ diabetes, (2) the combination of experimental periodontitis and insulin treatment affects the circulating biomarkers in $\mathrm{T} 1$ diabetes and (3) the addition of experimental periodontitis to experimentally-induced and insulin-treated $\mathrm{T} 1$ diabetes affects the circulating biomarkers.

The aim of the present study was to assess the effect of experimental periodontitis on the serum levels of leptin and IL-18 in rats with newly-induced and insulin-treated T1 diabetes.

\section{Materials and Methods}

\subsection{Animals}

Forty adult male Wistar rats (225-250 g) were used for the present randomized, parallel-arm 77 day experiment. The animals were randomly classified into two groups of 20 each: experimental diabetes and insulin administration (DI, control) and experimental diabetes, insulin administration and experimental periodontitis (DIP, control). The study followed the guidelines approved by the Council of the American Psychological Society (1980) and the European Communities Council Directive of 24 November 1996 (86/609/EEC). It was approved by the Ethics and Research
Committee of the National and Kapodistrian University of Athens and the Veterinary Directorate of the Prefecture of Athens.

Information on initial evaluation, housing and breding, diabetes induction, body weight and glucose evaluation, insulin administration, tooth ligation, histologic evaluation of the alveolar bone loss and sacrifice were analyzed in an earlier study involving the same animal sample. [23] In summary, a $45 \mathrm{mg} / \mathrm{kg} \mathrm{STZ}$ intravenous injection was used to induce diabetes. All rats received STZ injection on day 1. Successful diabetes induction was verified if serum glucose levels were $>300 \mathrm{mg} / \mathrm{dl}$ up to day 5 , measured with a glucometer. On day 5, insulin (Protaphane ${ }^{\circledR}$, Novo Nordisk $\mathrm{A} / \mathrm{S}$, Bagsværd, Denmark) treatment was initiated in all rats (subcutaneously, once/day). The dosage for each rat was adjusted daily. Periodontitis was induced at the maxillary right second molar in DIP rat group. Intrasulcular placement and ligation (ligation) of $4 / 0$ silk suture was used for periodontitis induction. The rats belonging to DIP group were kept ligated from day 16 (ligation day) and thereafter for 61 days. DI group was not ligated. All animals were sacrificed on day 77 (end of the study). The maxillary right second molar was studied histometrically for all animals. The presence of periodontitis was assessed histometrically based on the distance between the cemento-enamel junction and the alveolar crest mesially and distally of this tooth. [23] Serum glucose levels were assessed daily (77 glucose assessments per animal).

For each animal, two venous blood samples $(3.5 \mathrm{ml}$ each, after overnight fasting) were collected on days 16 and 77 , respectively. The blood samples were used to assess the serum levels of leptin and IL-18. Serum separator tubes were used and samples were left to clot for two hours at room temperature. Then centrifugation of the samples followed at $3000 \mathrm{rpm}$ for 15 minutes. Serum was removed, aliquoted and stored in Eppendorf tubes at $-80^{\circ} \mathrm{C}$. For each animal, serum levels of leptin and IL-18 were assessed on days 16 (baseline values) and 77 (final values).

\subsection{Biomedical Evaluation}

The analysis of the serum samples was performed by using multiplex immunoassay and read with Luminex 100 (Multiplexed Biomarker Immunoassays for Luminex ${ }^{\circledR}$ Instrumentation/xMAP ${ }^{\circledR}$ Technology-Luminex Corporation, Austin, USA). The levels of leptin and IL-18 were determined with MillipexTM Map Kit (Millipore Corporation, Billerica, MA, USA, Rat Cytokine/Chemokine, RCYTO-80K, RCYTO-80K-PMX). The animal was the unit of measurement for the biomarker values.

\subsection{Statistical Analysis}

For statistical analysis, mean values and standard deviations or median values and 1 and 3 quartiles (Q1-Q3) (when the normality assumption was not met) were calculated for serum leptin and IL-18 levels at two timepoints (day 16-baseline, day 77-final). The t-test or Mann-Whitney 
test for normally or non-normally distributed continuous variables was used for comparison of the above-mentioned measurements between the two study groups at each timepoint. Comparisons within groups between different timepoints were performed by t-test for dependent variables or the Wilcoxon matched pairs signed-ranks test. Statistical analysis was performed with STATA 9.1 (Stata, College Station, TX, USA). The level of statistical significance was set at $5 \%(\mathrm{p}=0.05)$.

\section{Results}

At the end of the study, 14 rats $(70 \%)$ in DI and 11 rats $(55 \%)$ in DIP group survived. Survival rate $(p=0.33)$, baseline and final weight and glycemic state prior to insulin onset were similar between groups. There was statistically significant weight gain at final evaluation for both groups. Diabetes was induced in all animals. Insulin treatment statistically significantly reduced glucose levels in both groups. Periodontitis was induced in all ligated rats. There was no statistically significant difference in median glucose levels between the groups on day 1 , on the day of diabetes confirmation and on days 16, 46, 61, 71 and 77. [23]

Baseline biomarker levels were similar between groups (table 1). At final evaluation, there were statistically significant differences between groups for IL-18 (MannWhitney $\mathrm{z}=2.08, \mathrm{p}=0.04$ ) (table 2). Comparison between final and baseline values for each biomarker and group showed that in DI final values as compared to baseline were statistically significantly higher for leptin and statistically significantly lower for IL-18 (table 3).

Comparison of the change in values (between final and baseline evaluation) for all biomarkers between groups revealed the following. Statistically significant difference in level change between the groups was found for IL-18. The median IL-18 change was negative for DI and positive for DIP. The absolute value of the median IL-18 change was statistically significantly higher for DI than DIP (table 4).

Table 1. Comparison of the baseline (at day 16) values of leptin and IL-18 between animal groups by using Mann-Whitney test.

\begin{tabular}{llll}
\hline Agent & Animal group & p-value \\
\hline & DI (n=14) & DIP (n=11) & \\
\hline Leptin & $1,976.9(954.2-5,265.7)$ & $1,461.4(621.7-2,773.3)$ & 0.55 \\
IL18 & $181.8(133.9-240.4)$ & $122.1(81.6-202.6)$ & 0.06 \\
\hline
\end{tabular}

DI: diabetes induction and insulin administration (control),

DIP: diabetes induction, insulin administration and periodontitis induction (test).

Table 2. Comparison of the final (at day 77) values of leptin and IL-18 between animal groups by using Mann-Whitney test.

\begin{tabular}{llll}
\hline Agent & Animal group & p-value \\
\hline & DI (n=14) & DIP (n=11) & \\
\hline Leptin & $3,491.8(2,338-5,946.6)$ & $3,176.6(755.6-4,366.9)$ & 0.38 \\
IL18 & $61.2(41.1-132.8)$ & $169.9(68.9-194.5)$ & 0.04 \\
\hline
\end{tabular}

DI: diabetes induction and insulin administration (control),

DIP: diabetes induction, insulin administration and periodontitis induction (test).
Table 3. Comparison between the final and baseline values of leptin and IL18 in each animal group by Wilcoxon matched pairs signed ranks tests.

\begin{tabular}{lll}
\hline Agent & Animal group & \\
\hline & DI & DIP \\
\hline $\mathbf{( p g / m I )}$ & $\mathbf{n}=\mathbf{1 4}$ & $\mathbf{n}=\mathbf{1 1}$ \\
\hline Leptin $_{\mathrm{b}}$ & $1,976.9(954.2-5,265.7)$ & $1,461.4(621.7-2,773.3)$ \\
Leptin $_{\mathrm{f}}$ & $3,491.8(2,338-5,946.6)$ & $3,176.6(755.6-4,366.9)$ \\
p-value & 0.01 & 0.09 \\
IL-18 $_{\mathrm{b}}$ & $181.8(133.9-240.4)$ & $122.1(81.6-202.6)$ \\
IL-18 $_{\mathrm{f}}$ & $61.2(41.1-132.8)$ & $169.9(68.9-194.5)$ \\
p-value & 0.01 & 0.59 \\
\hline
\end{tabular}

Leptin b, IL-18 b: Baseline (at day 16) values of leptin and IL-18, accordingly.

Leptin f, IL-18 f: Final values (at day 77) of leptin and IL-18, accordingly.

DI: diabetes induction and insulin administration (control),

DIP: diabetes induction, insulin administration and periodontitis induction (test).

Table 4. Comparison of the changes (between final and baseline evaluation) in leptin and IL-18 between animal groups by using Mann-Whitney tests.

\begin{tabular}{llll}
\hline Agent & Animal group & p-value \\
\hline $\mathbf{( p g} / \mathbf{m l})$ & DI $(\mathbf{n}=\mathbf{1 4})$ & DIP $(\mathbf{n}=\mathbf{1 1})$ & \\
\hline$\Delta$ Leptin & $916.8(26.6,2,718.1)$ & $2,179.7(110.5,2,485.2)$ & 1.00 \\
$\Delta$ IL18 & $-95.5(-143.3,-79.5)$ & $20.1(-53.2,92.7)^{\text {a }}$ & 0.009 \\
\hline
\end{tabular}

a statistically significant difference between groups DI and DIP (MannWhitney $\mathrm{z}=2.63, \mathrm{p}=0.009$ ).

$\Delta$ : change between final (day 77) and baseline (day 16) evaluation.

DI: diabetes induction and insulin administration (control),

DIP: diabetes induction, insulin administration and periodontitis induction (test).

\section{Discussion}

The present study compared serum leptin and IL-18 in the newly STZ-induced and insulin-treated $\mathrm{T} 1$ diabetes combined or not with ligature-induced periodontitis around one tooth. In the newly-induced and insulin-treated T1 diabetes, the continuation of insulin treatment for two more months significantly increased serum leptin levels and significantly reduced serum IL-18 levels. In the newlyinduced and insulin-treated T1 diabetes, the combination of the continuation of insulin treatment for two more months and the induction of periodontitis non-significantly increased serum leptin and IL-18 levels. In the newly-induced and insulin-treated $\mathrm{T} 1$ diabetes, the continuation of insulin treatment for two more months either combined or not to periodontitis increased serum leptin levels. The leptin increase was significant only in the absence of periodontitis. Serum IL-18 levels were significantly higher for the combination of periodontitis and insulin-treated T1diabetes as compared to insulin-treated $\mathrm{T} 1$ diabetes alone. The change in IL-18 levels was significantly lower and the change in leptin levels was non-significantly higher when the continuation of insulin treatment for two more months was combined with the induction of periodontitis as compared to the continuation of insulin treatment alone.

In newly STZ-induced T1 diabetes in rats, the present serum leptin levels were higher relatively long after insulin treatment initiation than soon after it. This finding is in agreement with results by Soliman [16] in newly STZ- 
induced $\mathrm{T} 1$ diabetes in rats, where serum leptin was higher at 21 than at 2 days of insulin treatment. Thus, in newly STZinduced diabetes in rats, the present study showed that the continuation of insulin treatment for two more months led to higher serum leptin at 72 (day 77) than at 11 days of insulin treatment (day 16), whereas Soliman's [16] study showed that the continuation of insulin treatment for 19 more days led to higher serum leptin at 21 than at 2 days of insulin treatment. Comparing study design between the present study and the study by Soliman, [16] it is similar for both studies concerning animal model, gender, diabetes induction method and insulin treatment and it differs in study duration. Specifically, the experiment ended at 77 days after STZ injection in this study and at 21 days after diabetes induction in Soliman's [16] study. The agreement in leptin findings between the two studies becomes even more important since they have similar study design.

In newly diagnosed children with $\mathrm{T} 1$ diabetes, serum leptin levels were significantly lower prior to insulin treatment initiation than 5 days after. In children with T1 diabetes receiving insulin treatment for a time period more than 2 years, the levels of serum leptin were significantly higher compared with those of age-matched healthy children. In undercontrolled diabetic children who had higher circulating hemoglobin A1c (HbA1c) concentrations and were then oversubstituted by insulin, leptin levels were elevated. [11]

The present increase in serum leptin levels with insulin treatment is explained by the fact that insulin stimulates leptin synthesis and secretion. [24] In this study, the increased insulin, as a result of insulin treatment, enhanced leptin secretion and led to elevated serum leptin. On the other hand, leptin plays important role in the regulation of glucose metabolism through food-intake independent mechanisms. [25] Leptin administration reduced hyperglycemia in mice with STZ-induced diabetes, independent of leptin signaling in the liver. [26]

The addition of periodontitis to insulin-treated diabetes led to non-significantly greater leptin increase (by 2.37 times). This might be explained through the link of leptin to inflammation. Leptin, as a proinflammatory cytokine, affects the secretion of acute-phase reactants, such as interleukin-1 (IL-1) and tumor-necrosis factor- $\alpha$ (TNF- $\alpha$ ), and promotes T helper 1 (Th1)-cell differentiation. Proinflammatory mediators that upregulate leptin expression, such as TNF- $\alpha$ and IL-1, help in turn in the creation of a loop of acute phase reactants that influence each other in promoting the development of chronic inflammation. [6] Serum leptin levels increased after exposure to inflammatory stimuli [4] and in experimental acute inflammation. Serum leptin was associated with inflammatory biomarkers [6] and was positively related to the active phase of rheumatoid arthritis. [7] In the periodontal tissues, leptin upregulates the synthesis of proinflammatory and proteolytic molecules [8]. In humans, serum leptin was significantly elevated in periodontitis [27] and positively related to the severity of periodontal loss. [28] Serum leptin in non-obese individuals was significantly elevated in periodontitis, as assessed in a systematic review and meta-analysis. [29]

Serum IL-18 levels were significantly higher in mice with experimentally-induced $\mathrm{T} 1$ diabetes [17] and in $\mathrm{T} 1$ diabetes patients as compared to controls. [29-31] Despite the IL-18 increase in T1 diabetes, the serum levels of IL-18 binding protein (IL-18BP) and IL-37, which are negative regulators of IL-18 function, were similar for T1 diabetic individuals and controls. [30] On the other hand, there was positive correlation between serum IL-18 and IL-18BP levels and significant increase of the free, unbound IL-18 in T1 diabetes. [31] Serum IL-18 was higher in T1 diabetes patients with poor than good glycemic control. [29-31] Specifically, serum IL-18 levels in T1 diabetes patients were positively correlated with glucose levels [28] and with HbAlc. [30, 31] Serum IL-18BP levels were positively correlated to HbA1c levels in T1 diabetes patients. [31] Moreover, the serum IL18 levels were significantly elevated in early $\mathrm{T} 1$ diabetes stages in humans, [32] where proper glycemic control might have not been achieved. These findings reveal a link between IL-18 and glycemic control in T1 diabetes. In the present study, in the newly-induced $\mathrm{T} 1$ diabetes the continuation of insulin treatment for two more months led to reduction of the glucose levels. The present significant reduction in serum IL18 levels with the continuation of insulin treatment is explained by the reduced glucose levels achieved with insulin treatment.

The addition of periodontitis to insulin-treated diabetes changed the type of effect exerted on serum IL-18 levels by increasing them. In the combined insulin-treated diabetes and periodontitis group, the non-significant increase in IL-18 with time and the significantly higher IL-18 as compared to insulin-treated diabetes alone might be attributed to the ligation-induced inflammation. Periodontitis patients had increased IL-18 [18] and periodontitis was positively associated with IL-18 levels in patients planned for diagnostic coronary angiography. [33] IL-18 is implicated in the pathogenesis of inflammatory diseases, [31] enhances immune reactions related to both Th1 and T helper 2 (Th2)cells and enhances the superiority of Th1 over Th2 cells. [34] The balance between the immune reactions related to Th1 cells and those related to Th2 cells is important in the pathogenesis of periodontitis. The present findings might be the result of synergistic and antagonistic effects among the various mediators of inflammation in periodontitis. The present IL-18 change observed was significantly greater (by 4.75 times) for the insulin-treated diabetes alone than for the combination of insulin-treated diabetes and periodontitis. The type of IL-18 change differed between the two groups, which partly explains this finding. It seems that the effect of insulintreated diabetes on IL-18 was stronger than the effect of the combination of insulin-treated diabetes and periodontitis. The addition of periodontitis alleviated the effect of insulintreated diabetes on IL-18.

In summary, the present study in rats found the following. In the newly-induced $\mathrm{T} 1$ diabetes the continuation of insulin treatment for two more months induced changes in the levels 
of circulating biomarkers implying regulation of the glycose levels. The addition of periodontitis to newly-induced and insulin-treated $\mathrm{T} 1$ diabetes induced changes in the levels of circulating biomarkers suggestive of alterations in the systemic inflammation generated by insulin treated $\mathrm{T} 1$ diabetes.

The present findings should be interpreted with caution since periodontitis was induced around one tooth, which might have affected the level of systemic inflammation, the number of animals studied was relatively small and the findings might differ in naturally occurring diabetes and periodontitis in humans. In future studies another time point of the assessment of the biomarkers might be added, specifically the day of diabetes induction. The three time points of biomarkers assessment would allow intragroup comparisons before insulin treatment initiation, soon after it and relatively long after it.

\section{Conclusion}

Within its limits, this study in rats showed that experimental periodontitis induced changes in serum biomarker levels suggestive of alterations in the systemic inflammation generated by insulin-treated $\mathrm{T} 1$ diabetes.

\section{Statement of Any Potential Source of Funding and Conflict of Interest}

The authors declare that they have no conflicts of interest and that no funding was used for this study.

\section{References}

[1] American Diabetes Association Diagnosis and classification of diabetes mellitus. Diabetes Care. 2014; 37 (Suppl. 1): S81S90.

[2] Papapanou PN, Sanz M, Buduneli N, Dietrich T, Feres M, Fine DH et al. Periodontitis: Consensus report of workgroup 2 of the 2017 World Workshop on the Classification of Periodontal and Peri-Implant Diseases and Conditions. J Periodontol. 2018; 89 (Suppl 1): S173-S182.

[3] Sanz M, Ceriello A, Buysschaert M, Chapple I, Demmer RT, Graziani $\mathrm{F}$ et al. Scientific evidence on the links between periodontal diseases and diabetes: Consensus report and guidelines of the joint workshop on periodontal diseases and diabetes by the International Diabetes Federation and the European Federation of Periodontology. J Clin Periodontol. 2018; 45: 138-149.

[4] Chapple ILC, Genco R and on behalf of working group 2 of the joint EFP/AAP workshop. Diabetes and periodontal diseases: consensus report of the Joint EFP/ AAP Workshop on Periodontitis and Systemic Diseases. J Clin Periodontol. 2013; 40 (Suppl. 14): S106-S112.

[5] Landman RE, Puder JJ, Xiao E, Freda PU, Ferin M, Warldaw SL. Endotoxin stimulates leptin in the human and nonhuman primate. J Clin Endocrinol Metab. 2003; 88 (3): 1285-1291.
[6] Iikuni N, Lam QLK, Lu L, Matarese G, La Cava A. Leptin and Inflammation. Curr Immunol Rev. 2008; 4 (2): 70-79.

[7] Targońska-Stepniak B, Majdan M, Dryglewska M. Leptin serum levels in rheumatoid arthritis patients: relation to disease duration and activity. Rheumatol Int. 2008; 28: 585-591.

[8] Deschner J, Eick S, Damanaki A, Nokhbehsaim M. The role of adipokines in periodontal infection and healing. Mol Oral Microbiol. 2014; 29 (6): 258-269.

[9] Zhu J, Guo B, Gan X, Zhang L, He Y, Liu B et al. Association of circulating leptin and adiponectin with periodontitis: a systematic review and meta-analysis. BMC Oral Health. 2017; 17 (1): 104-118.

[10] Zekeridou A, Mombelli A, Cancela J, Courvoisier D, Giannopoulou C. Systemic inflammatory burden and local inflammation in periodontitis: What is the link between inflammatory biomarkers in serum and gingival crevicular fluid? Clin Exp Dent Res. 2019; 5 (2): 128-135.

[11] Soliman AT, Omar M, Assem HM, Nasr IS, Rizk MM, Matary WEI et al. Serum leptin concentrations in children with type 1 diabetes mellitus: relationship to body mass index, insulin dose, and glycemic control. Metabolism. 2002; 51 (3): 292-296.

[12] Dogan Y, Akarsu S, Ustunday B, Yilmaz E, Gurgoze MK. Serum IL-1beta, IL-2, and IL-6 in insulin dependent diabetic children. Mediators Inflamm. 2006; 1 (59206): 1-6.

[13] Stechova K, Bohmova K, Vrabelova Z, Sepa A, Stadlerova G, Zacharovova $\mathrm{K}$ et al. High T-helper-1 cytokines but low Thelper-3 cytokines, inflammatory cytokines and chemokines in children with high risk of developing type 1 diabetes. Diabetes Metab Res Rev. 2007; 23: 462-471.

[14] Buduneli N and Kinane DF. Host-derived diagnostic markers related to soft tissue destruction and bone degradation in periodontitis. J Clin Periodontol. 2011; 38 (Suppl 11): 85-105.

[15] Takeda M, Ojima M, Yoshioka H, Inaba H, Kogo M, Shizukuishi S et al. Relationship of serum advanced glycation end products with deterioration of periodontitis in type 2 diabetes patients. J Periodontol. 2006; 77: 15-20.

[16] Soliman NA. Effect of experimentally induced diabetes mellitus on serum leptin level and the role of insulin replacement therapy. Egypt J Hosp Med. 2001; 3: 190-208.

[17] Rothe H, Jenkins NA, Copeland NG, Kolb H. Active stage of autoimmune diabetes is associated with the expression of a novel cytokine, IGIF, which is located near IDD2. J Clin Invest. 1997; 99 (3): 469-474.

[18] Sánchez-Hernández PE, Zamora-Perez AL, Fuentes-Lerma M, Robles-Gómez C, Mariaud-Schmidt RP, Guerrero-Velázquez C. IL-12 and IL-18 levels in serum and gingival tissue in aggressive and chronic periodontitis. Oral Dis. 2011; 17 (5): $522-529$.

[19] Ghanim H, Korzeniewski K, Sia CL, Abuaysheh S, Lohano T, Chaudhuri A, Dandona P. Suppressive effect of insulin infusion on chemokines and chemokine receptors. Diabetes Care. 2010; 33 (5): 1103-1108.

[20] Holzhausen M, Garcia DF, Pepato MT and Marcantonio EJr. The influence of short-term diabetes mellitus and insulin therapy on alveolar bone loss in rats. J Periodont Res. 2004; 39 (3): 188-193. 
[21] Cetinkaya BO, Keles GC, Ayas B, Sakallioglu EE and Acikgoz G. The expression of vascular endothelial growth factor in a rat model at destruction and healing stages of periodontal disease. J Periodontol. 2007; 78 (6): 1129-1135.

[22] Pepelassi E, Xynogala I, Perrea D, Agrogiannis G, Pantopoulou A, Patsouris E et al. Histometric assessment of the effect of diabetes mellitus on experimentally induced periodontitis in rats. J Int Acad Periodontol. 2012; 14 (2): 3541.

[23] Xynogala I, Pepelassi E, Perrea D, Agrogiannis G, Pantopoulou A, Patsouris E et al. Adiponectin and interleukin6 levels in insulin-treated diabetic rats with experimental periodontitis. Braz Oral Res. 2012; 26 (1): 71-76.

[24] Amitani M, Asakawa A, Amitani H, Inui A. The role of leptin in the control of insulin-glucose axis. Front Neurosci. 2013; Apr 8, doi: 10.3389/fnins.2013.00051.

[25] Kim GH, Szabo A, King EM, Ayala J, Ayala JE, Altarejos JY. Leptin recruits Creb-regulated transcriptional coactivator 1 to improve hyperglycemia in insulin-deficient diabetes. Mol Metab. 2014; 4 (3): 227-236.

[26] Denroche HC, Levi J, Wideman RD, Sequeira RM, Huynh FK, Covey SD et al. Leptin therapy reverses hyperglycemia in mice with streptozotocin-induced diabetes, independent of hepatic leptin signaling. Diabetes. 2011; 60 (5): 1414-1423.

[27] Shimada Y, Komatsu Y, Ikezawa-Suzuki I, Tai H, Sugita N, Yoshie H. The effect of periodontal treatment on serum leptin, interleukin-6, and C-reactive protein. J Periodontol. 2010;81 (8): 1118-1123.
[28] Karthikeyan BV and Pradeep AR. Gingival crevicular fluid and serum leptin: their relationship to periodontal health and disease. J Clin Periodontol. 2007; 34 (6): 467-472.

[29] Altinova AE, Yetkin I, Akbay E, Bukan N, Arslan M. Serum IL-18 levels in patients with type 1 diabetes: relations to metabolic control and microvascular complications. Cytokine. 2008; 42 (2): 217-221.

[30] Ryba-Stanisławowska M, Rybarczyk-Kapturska K, Myśliwiec M, Myśliwska J. Elevated Levels of Serum IL-12 and IL-18 are Associated with Lower Frequencies of CD4+ CD25highFOXP3 + Regulatory T cells in Young Patients with Type 1 Diabetes. Inflammation. 2014; 37 (5): 1513-1520.

[31] Harms RZ, Yarde DN, Guinn Z, Lorenzo-Arteaga KM, Corley $\mathrm{KP}$, Cabrera MS et al. Increased expression of IL-18 in the serum and islets of type 1 diabetics. Mol Immunol. 2015; 64 (2): 306-312.

[32] Nicoletti F, Conget I, Di Marco R, Speciale AM, Morìnigo R, Bendtzen $\mathrm{K}$ et al. Serum levels of the interferon-gammainducing cytokine interleukin-18 are increased in individuals at high risk of developing type 1 diabetes. Diabetologia. 2001; 44 (3): 309-311.

[33] Schallhorn RA, Patel DN, Chandrasekar B, Mealey BL. Periodontal disease in association with systemic levels of Interleukin-18 and CXC ligand 16 in patients undergoing cardiac catheterization. J Periodontol. 2010; 81 (8): 1180-1186.

[34] Orozco A, Gemmell E, Bickel M, Seymour GJ. Interleukin1beta, interleukin-12 and interleukin-18 levels in gingival fluid and serum of patients with gingivitis and periodontitis. Oral Microbiol Immunol. 2006; 21 (4): 256-260. 